\title{
A Propensity Score Matching study on the effect of OnabotulinumtoxinA alone versus Short-Term Psychodynamic Psychotherapy plus drug-of-choice as preventive therapy in Chronic Migraine: effects and predictive factors.
}

\section{Michele Alessiani}

Universita Campus Bio-Medico di Roma

\section{Barbara Petolicchio}

Universita degli Studi di Roma La Sapienza

\section{Rita De Sanctis}

Humanitas University

Romina Di Giambattista

IREP

\section{Marta Puma}

Universita degli Studi di Roma La Sapienza

\section{Ciro Franzese}

Humanitas University

Edmond Gilliéron

IREP

Alessandro Viganò ( $\square$ alessandro.vigano@uniroma1.it )

Sapienza - University of Rome https://orcid.org/0000-0002-8079-5354

\section{Vittorio Di Piero}

Universita degli Studi di Roma La Sapienza

Research article

Keywords: headache, migraine, onabotulinumtoxinA, psychotherapy, Medication Overuse Headache

Posted Date: April 13th, 2020

DOI: https://doi.org/10.21203/rs.3.rs-21658/v1

License: (1) (1) This work is licensed under a Creative Commons Attribution 4.0 International License. Read Full License 


\section{Abstract}

Background: Chronic migraine (CM) is an ensemble disorder in which pain, comorbidities, and medication overuse headache $(\mathrm{MOH})$ interact to determine the degree of disability. No data support the choice of a monotherapy rather than a multidisciplinary preventive approach. The aim of this study is testing the superiority of multidisciplinary approach, i.e. Short-term Psychodynamic Psychotherapy (STPP) plus drug of choice, vs. monotherapy, i.e. OnabotulinumtoxinA.

Methods: We consecutively recorded data from CM patients who underwent STPP or OnaBoNT-A, with a 3-month follow-up schedule. Headache days and analgesics intake were monitored as primary outcome measures to determine the benefit. Propensity score matching (PSM) was used to eliminate discrepancies between groups. Discriminant function analysis was used to pinpoint predictive factors associated to the clinical response.

Results: 96 patients with $\mathrm{CM}$ (64\% with $\mathrm{MOH})$ were treated with STPP and 54 (59\% with $\mathrm{MOH})$ with OnaBoNT-A. At baseline, OnaBoNT-A patients had more failed preventive therapies, more years of illness and chronicity, and were older, while STPP patients were more depressed and had an higher HIT-6 $(p<0.001)$. Both STPP and OnaBoNT-A patients showed a significant reduction of headache days (STPP: -14 vs. OnaBoNT-A:-14.3) and analgesics intake (STPP: -12,3 vs. OnaBoNT-A -13.5 pills/month), respectively. $\mathrm{MOH}$ diminished more in STPP, adherence was higher in OnaBoNT-A. Results were confirmed after that PSM balanced the two groups for all these variables that resulted different (but age). DFA predicts responders in pooled and OnaBoNT-A group.

Conclusions: OnaBoNT-A monotherapy produced similar results of a multidisciplinary approach with psychotherapy plus medication. STPP provided a better short term response but short- and long-term adherence was higher in OnaBoNT-A.

\section{Background}

Chronic migraine $(\mathrm{CM})$ patients, with or without Medication Overuse Headache $(\mathrm{MOH})$, represent the larger share of migraine patients referring to specialized headache clinics [1-2]. These CM patients are difficult to treat, and only one out of five comply with preventive treatment and less than half clinically improve [3].

CM is difficult to manage because it is a complex "ensemble" of disorders rather than a single entity. Many somatic (e.g. asthma, obesity) and psychiatric (e.g. anxiety, depression, bipolar disorder) comorbidities concur to draw, together with chronic headache, a personalized pattern of disability in these patients [4].

The relationship between pain and comorbidities is, in fact, bidirectional and intricate, since the presence of one can increase the odd of having the others and vice versa. For example, on one hand, depression 
increases the risk of migraine chronicization [5], as well as stressful events and certain personalities [6], but, on the other hand, chronic pain worsens depressive symptoms [7].

Therefore, the choice of the best therapeutic option for these patients is difficult, and patients could benefit from a global approach more than treating their comorbidities individually. As a rule of thumb, a multidisciplinary approach is often used and it is not uncommon to take care of psychiatric comorbidities or improve stress management, concomitantly with pharmacological prevention of migraine $[8,9]$.

Besides making the burden of $\mathrm{CM}$ heavier, these comorbidities can also reduce the chance of therapeutic success in other ways. For example, comorbidities may contraindicate the use of the certain preventive migraine medications due to their side effects (e.g. beta-blockers in depressed patients) or drug-drug interaction with medications used for the treatment of comorbidity itself [10]. Non-pharmacological or pharmacological non-interacting interventions are therefore extremely useful in CM management.

An interesting non-pharmacological option is psychotherapeutic support, such as the short-term psychodynamic psychotherapy (STPP) [11] or the mindfulness-based intervention [12]. Our group previously demonstrated that the STPP, a form of psychotherapy proposed by Edmond Gilliéron and widely used in the treatment of several psychiatric diseases [13-15], reduced headache days and decreased the chance of relapse into medication overuse, when added to pharmacological therapy in $\mathrm{MOH}$ patients [11].

In the view of avoiding drug-drug interactions also the treatment with OnabotulinumtoxinA (OnaBoNT-A) is particularly important. OnaBoNT-A has specifically been approved as preventive therapy of CM in adults, after the results obtained by two large multicenter randomized clinical trials - Phase III Research Evaluating Migraine Prophylaxis Treatment (PREEMPT) 1 and $2[16,17]$. Several post-marketing research has found that a good share of patients with CM maintain their response also in the following years of treatment [18-20]. Interestingly, recent evidence showed that the treatment with OnaBoNT-A also improved depression and anxiety in CM patients [21], with a greater benefit in those patients that showed an improvement of pain symptoms.

Due to the possible overlapping effects provided by OnaBoNT-A or a multidisciplinary approach, in the present study we tested the hypothesis that a multidisciplinary (drug-of choice primed by STTP) approach can be superior to only OnaBoNT-A.

\section{Material And Methods}

\section{Patients' recruitment and baseline evaluation}

We consecutively recruited all adult patients (age > 18 years) affected by $\mathrm{CM}$ with and without $\mathrm{MOH}$ (ICHD-III 1.3 and 8.2) who were considered to start STPP or OnaBoNT-A as preventive treatment in the Headache Center at the Department of Human Neurosciences, Sapienza - University of Rome. According to the pragmatic nature of the study, we kept exclusion criteria to a minimum: we excluded patients 
without a clear headache diagnosis or the coexistence of two different headaches (except $\mathrm{MOH}$ ), and with other severe neurological or psychiatric diseases. We also excluded patients with contraindications to perform OnaBoNT-A (e.g. neuromuscular junction disease, skin infections or diseases in the head and/or neck, allergy to OnaBoNT-A, use of anticoagulant therapy). We considered as screening failure those patients that interrupted treatment after the first administration of OnaBoNT-A or did not finish the first phase of STPP (Brief Psychodynamic Interview meetings, see below). After that point, patients who quitted the study, were considered as dropouts and considered in the analysis. All patients underwent neurological examination and, when appropriate, instrumental investigations (e.g. brain neuroimaging, electroencephalogram).

At baseline, we recorded demographic and headache characteristics (see Table 1) as well as migraine history (both years since migraine onset and years since the migraine chronicity). As primary outcomes, we recorded the number of monthly headache days, and the use of analgesics. Validated scales for disability (HIT-6 and MIDAS) and psychiatric comorbidities (Hamilton Rating Scale for Depression and Anxiety) were recorded at baseline and during follow-up visits. Patients were divided in three categories of response according to practical criteria: complete response (CR, patients who remitted to a low-frequency episodic migraine not needing preventive therapy, i.e. less than 5 headache days/month), partial response (PR, patients reverting from CM to EM but requiring further preventive treatment, 5 to 14 headache days/month) and no response (NR, patients who remained with $\mathrm{CM}$ ). The study was approved by our Ethic Committee within the context of a larger study on predictive factors of response to migraine preventive therapies, and all patients participating in the study were asked to sign a written informed consent for the use of their clinical data. The study was conducted according to the Declaration of Helsinki. 
Table 1

Baseline demographic and clinical features of the OnaBoNT-A and STPP group at baseline. Significant differences are marked in bold. Data are presented with mean and standard deviation. While age still remains significantly different $(p=0.03)$, the other parameters were all paired after trimming.

\begin{tabular}{|lllll|}
\hline & OnaBoNT-A $(\boldsymbol{n}=\mathbf{5 4})$ & STPP $(\boldsymbol{n}=\mathbf{9 6})$ & p-value & p-value after PSM \\
\hline Age & $54.75( \pm 15.08)$ & $41.31( \pm 13.05)$ & $p<0.001$ & $p=0.03$ \\
\hline School years & $12.16( \pm 4.53)$ & $13.23( \pm 3.69)$ & $p=0.121$ & \\
\hline Headache years & $34.16( \pm 16.59)$ & $23.71( \pm 12.93)$ & $p<0.001$ & $p=0.29$ \\
\hline Chronicization years & $11.63( \pm 10.47)$ & $6.10( \pm 7.31)$ & $p<0.001$ & $p=0.47$ \\
\hline HIT-6 & $56.84( \pm 9.20)$ & $64.66( \pm 8.29)$ & $p<0.001$ & $p=0.13$ \\
\hline MIDAS & $52.03( \pm 47.79)$ & $55.33( \pm 49.80)$ & $p=0.695$ & \\
\hline HAM-D & $9.56( \pm 7.99)$ & $16.80( \pm 9.01)$ & $p<0.001$ & $p=0.28$ \\
\hline HAM-A & $14.75( \pm 7.95)$ & $17.66( \pm 9.52)$ & $p=0.061$ & \\
\hline HA days & $25.47( \pm 6.25)$ & $23.70( \pm 6.40)$ & $p=0.107$ & \\
\hline$N^{\circ}$ analgesics & $20.24( \pm 22.10)$ & $17.12( \pm 15.16)$ & $p=0.311$ & \\
\hline
\end{tabular}

\section{Study design}

STPP was proposed as initial approach to first-visit patients according to their profile. Patients were instructed about STPP (see below for details) and about the fact that, in case of failure of STPP, a personalized pharmacological preventive therapy would have been added. On the other hand, OnaBoNT-A was proposed to patients fulfilling Italian Agency for Drugs criteria for the use of OnaBoNT-A (i.e. failure of at least two preventive treatments). All patients were instructed to fill the monthly headache diary and record the number of acute headache medications used. Clinical evaluation of patients was performed every 3 months, recording monthly headache days and number of acute headache medications used each month, according to our standard and to the PREEMPT protocol.

\section{OnaBoNT-A arm}

OnaBoNT-A was administered following the PREEMPT protocol in 31 sites of head-neck district, $5 \mathrm{UI}$ for each site with a total of $155 \mathrm{UI}[16,17]$. OnaBoNT-A was administered every 12 weeks for 5 sessions. No other preventive therapy was added during these 5 administrations. The starting dose was always $155 \mathrm{UI}$ and, when appropriate, the dosage of OnaBoNT-A was increased according to the "follow the pain" protocol (195 UI).

\section{STPP arm}


Patients underwent a two-phase psychotherapy [22, 23]. The first phase, the Brief Psychodynamic Interview (BPI), is mostly diagnostic and it is developed during the first four meetings. The object of BPI is to identify the inner conflict of the patient and is conducted using associative anamnesis and initial interpretation techniques. Therapeutic STPP is delivered during a second phase of eight meetings (over 60 days). STPP is inspired by Freudian technique (psychoanalyst's neutral attitude, free associations, free-floating attention), but it also relies on contextual modifications occurring in the face-to-face psychoanalytic setting (transference and counter-transference). In its main core, STPP directly aims at a central psychological problem to help patients recognize it and, possibly, solve it. During the BPI, if the patient resists the attempt to identify the inner conflict, or instead, reaches its complete comprehension, the psychotherapy is interrupted. At the first follow-up visit (90 days), all patients who didn't reach a CR started a standard preventive pharmacological therapy, while CR patients continued clinical follow-up. From the second follow-up visit (180 days), therapies were increased or substituted if needed, otherwise they were stopped if patients reached CR according to the treating physician's choice.

\section{Statistical Analysis}

The variables distribution has been analyzed with the Shapiro-Wilk's test for normality. Parametric and nonparametric tests were used accordingly. Continuous baseline data were analyzed with ANOVA with post-hoc comparison between groups. Categorical baseline data were analysed with Chi-squared test with Yates' correction. We used repeated measures ANOVA for evaluating the time-depending dynamics of groups. Considering dropouts, within and in-between group results were controlled by post-hoc comparison with Bonferroni correction. In order to compare the size of the response between groups, the Propensity Score Matching (PSM) was used for a second analysis. PSM is a statistical analysis that mimics randomization, since it reestablishes balance between groups according to the propensity of individuals to be treated in a certain way. PSM pairs observations (matches) from groups and trimmed not-matched cases, i.e. patients with an absolute standard bias $>0.20$. PSM was implemented by a logistic regression approach [24-26]. Baseline variables with a significant difference between groups were chosen as parameters on which to perform the matching rather than using an aprioristic approach.

We used Discriminant Function Analysis (DFA) to identify factors associated to early ( 90 days) and late (360 days) response. DFA estimates the linear combination of covariates separating individuals according to an outcome of interest, in our case the response category (CR, PR, NR) [27-30]. Models were calculated for both early (at 90 days) and late (at the end of the 1-year follow-up) response. The approach and calculation parameters were based on previous papers [28-30]. The resulting model presents the input variables in terms of their own weight in the prediction (i.e. the higher the value of the Partial Lambda, the more prominent the role). DFA was performed on the pooled sample and separately within the two groups.

\section{Results}




\section{Real-world comparison between OnaBoNT-A and STPP plus pharmacological therapy}

We consecutively recruited $152 \mathrm{CM}$ patients who underwent STPP or OnaBoNT-A treatment. Two patients resulted as screening failures; therefore, 150 patients were considered for the analysis. Fifty-four patients were treated with OnaBoNT-A (59\% with also $\mathrm{MOH}$ ) and 96 with STPP (64\% with also $\mathrm{MOH}$ ) (see Table 1$)$.

Repeated-measure ANOVA showed a progressive reduction of headache days from 0 to 360 days $(F=$ $27.6, p<0.0001)$ with no global difference between groups $(F=2.6 ; p=0.11)$. Time-Group interaction was similar for both groups $(F=0.85 ; p=0.49)$ (see Table 2, Fig. $1 A)$.

Table 2

Original Analysis (OA) and after propensity score trimming (AT). Group differences in the number of headache days and analgesics consumption. Significant differences (after Bonferroni correction) between groups are marked in bold. After the trimming, the analysis confirmed the results found in the original analysis.

\begin{tabular}{|c|c|c|c|c|c|c|}
\hline Baseline & 90 days & 180 days & 270 days & $\begin{array}{l}365 \\
\text { days }\end{array}$ & & \\
\hline \multirow[t]{3}{*}{$\begin{array}{l}\text { Headache } \\
\text { Days }\end{array}$} & $\begin{array}{l}\text { STPP (OA) } \\
\text { OnaBoNT-A } \\
(\text { OA) }\end{array}$ & $\begin{array}{l}23.7 \pm \\
6.4 \\
25.5 \pm \\
6.2\end{array}$ & $\begin{array}{l}16.5 \pm \\
9.7 \\
18.6 \pm \\
9.4\end{array}$ & $\begin{array}{l}15.3 \pm \\
8.8 \\
19.6 \pm \\
9.3\end{array}$ & $\begin{array}{l}12.9 \pm \\
8.4 \\
14.7 \pm \\
9.6\end{array}$ & $\begin{array}{l}9.7 \pm 8.3 \\
11.2 \pm \\
8.9\end{array}$ \\
\hline & STPP (AT) & $\begin{array}{l}24.63 \pm \\
6.1\end{array}$ & $\begin{array}{l}15.0 \pm \\
10.8\end{array}$ & $\begin{array}{l}13.7 \pm \\
9.9\end{array}$ & $\begin{array}{l}11.9 \pm \\
8.3\end{array}$ & $\begin{array}{l}10.8 \pm \\
9.4\end{array}$ \\
\hline & $\begin{array}{l}\text { OnaBoNT-A } \\
\text { (AT) }\end{array}$ & $\begin{array}{l}25.6 \pm \\
6.5\end{array}$ & $\begin{array}{l}19.5 \pm \\
9.4\end{array}$ & $\begin{array}{l}20.7 \pm \\
9.2\end{array}$ & $\begin{array}{l}16.3 \pm \\
10.3\end{array}$ & $\begin{array}{l}11.9 \pm \\
10.2\end{array}$ \\
\hline \multirow[t]{3}{*}{$\begin{array}{l}\text { Analgesics } \\
\text { Consumption }\end{array}$} & $\begin{array}{l}\text { STPP (OA) } \\
\text { OnaBoNT-A } \\
(\text { OA) }\end{array}$ & $\begin{array}{l}17.2 \\
\pm 15.2 \\
20.1 \pm \\
22.1\end{array}$ & $\begin{array}{l}6.9 \pm 6.4 \\
11.1 \pm \\
10.3\end{array}$ & $\begin{array}{l}7.1 \pm \\
6.3 \\
11.9 \pm \\
9.6\end{array}$ & $\begin{array}{l}6.7 \pm 6.6 \\
8.9 \pm 8.5\end{array}$ & $\begin{array}{l}4.9 \pm 4.9 \\
6.6 \pm 6.2\end{array}$ \\
\hline & STPP (AT) & $\begin{array}{l}17.3 \pm \\
12.5\end{array}$ & $6.4 \pm 9.8$ & $\begin{array}{l}6.8 \pm \\
7.0\end{array}$ & $7.4 \pm 9.2$ & $5.0 \pm 7.2$ \\
\hline & $\begin{array}{l}\text { OnaBoNT-A } \\
\text { (AT) }\end{array}$ & $\begin{array}{l}19.1 \pm \\
22.1\end{array}$ & $\begin{array}{l}10.5 \pm \\
9.8\end{array}$ & $\begin{array}{l}12.2 \pm \\
9.7\end{array}$ & $9.9 \pm 9.2$ & $6.6 \pm 6.5$ \\
\hline
\end{tabular}


Table 3

A-B-C. Results on the weight of each variable from DFA analysis on prediction. A. Pooled data. B. OnaBoNT-A. C. STPP. In bold the significant parameters within each model.

\begin{tabular}{|c|c|c|c|c|c|c|c|c|}
\hline A. Pooled & Partial & $\begin{array}{l}\mathrm{p}- \\
\text { level }\end{array}$ & $\begin{array}{l}\text { B. OnaBoNT- } \\
\text { A }\end{array}$ & Partial & $\begin{array}{l}\mathrm{p}- \\
\text { level }\end{array}$ & C. STPP & Partial & $\begin{array}{l}\text { p- } \\
\text { level }\end{array}$ \\
\hline HA days & 0.930 & 0.007 & HA days & 0.853 & 0.035 & HA days & 0.956 & 0.140 \\
\hline HIT-6 & 0.935 & 0.010 & HAM-A & 0.892 & 0.091 & HAM-A & 0.942 & 0.074 \\
\hline HAM-A & 0.944 & 0.020 & HIT-6 & 0.784 & 0.006 & $\begin{array}{l}\mathrm{N}^{\circ} \\
\text { analgesics }\end{array}$ & 0.969 & 0.250 \\
\hline Curr. Proph. & 0.954 & 0.041 & HAM-D & 0.830 & 0.020 & $\begin{array}{l}\text { Curr. } \\
\text { Prophyl. }\end{array}$ & 0.972 & 0.294 \\
\hline \multirow[t]{2}{*}{$\begin{array}{l}\text { School } \\
\text { years }\end{array}$} & 0.977 & 0.210 & $\begin{array}{l}\mathrm{N}^{\circ} \\
\text { analgesics }\end{array}$ & 0.880 & 0.068 & HIT-6 & 0.975 & 0.329 \\
\hline & & & & & & $\begin{array}{l}\text { School } \\
\text { years }\end{array}$ & 0.977 & 0.358 \\
\hline
\end{tabular}

At 90 days, 8 STPP patients became complete responders (CR) and continued only with follow-up visits, 88 added a preventive therapy. At the same time, 4 OnaBoNT-A patients became CR, while 50 maintained $\mathrm{CM}$ or reversed to PR. Despite the small number of CR in both groups, the larger drop in mean number of headache days per month is observed at this point with both OnaBoNT-A patients (-6.9 headache days/month) and STPP (-9.4 headache days/month) compared to baseline (see Table 2).

The post-hoc comparison showed that the two groups only differed at 180 days follow-up with a better improvement in STPP (-8.4 headache days/month) vs. OnaBoNT-A (-5.9 headache days/month) ( $p=0.04$ after correction). At 360 days, STPP group reached on average -14 headache days/month while OnaBoNT-A group had on average -14.3 days/month. At 360 days, both groups had a significant reduction compared to baseline (after post-hoc comparison with Bonferroni correction: $p<0.0001$ for both groups) but the difference was not significant between groups (see Table 2).

The intake of acute headache medications also decreased overtime $(F=9.65 ; p<0.0001)$ with no significant global different between groups $(F=1.19 ; p=0.28)$ nor in the Time-Group interaction $(F=0.19$; $\mathrm{p}=0.94$ ) (see Fig. 1B and Table 2). STPP patients had a larger decrease at 90 and 180 days (see Fig. 1B). Considering the interruption of $\mathrm{MOH}$, we found that the number of $\mathrm{MOH}$ patients significantly decreased in the STPP compared to OnaBoNT-A group at 3 months $(p<0.001)$.

In the STPP group, the HAM-D improved significantly from baseline $(16.8 \pm 9.01)$ to 90 days $(13.3 \pm 8.7)$ $(p=0.01)$, while it didn't occur in OnaBoNT-A group. The HAM-A didn't differ in the two groups at any time.

In the OnaBoNT-A group, 23 out of 54 completed the 1-year follow-up. From the original sample, 15 (28\%) achieved a complete response, $3(5.6 \%)$ had a partial response, and $5(9.2 \%)$ maintained CM. In the STPP 
group, out of 96 patients, 21 reached the 12-month evaluation. In 17 (18.1\%) patients, a complete response was achieved, $3(3.2 \%)$ patients had a partial response and $1(1.1 \%)$ patient remained in CM.

The overall dropout rate at 12 months was $78 \%$ in the STPP group and 55\% in the OnaBoNT-A group. The rate of dropout was higher in the STPP than in OnaBoNT-A group $(p=0.017)$. In the OnaBoNT-A group, adherence was $100 \%$ at $90,87 \%$ at $180,65 \%$ at 270 , and $55 \%$ at 360 days. In the STPP group adherence was $100 \%$ at $90,72 \%$ at $180,72 \%$ at 270 , and dropped to $21 \%$ at 360 days. The difference in the adherence between OnaBoNT-A and STPP was also significant at 180 days ( $87 \%$ vs. $72 \%, p=0.007)$.

\section{Baseline group differences and propensity score}

At baseline, OnaBoNT-A patients were older, with more years of migraine and more years of chronicization than STPP patients. By contrast, STPP patients had a higher score on HIT-6 and HAM-D scales (ANOVA F $=10.8, p<0.001)$. All these parameters resulted significantly different after post-hoc comparison.

Complete baseline clinical and demographic details of STPP and OnaBoNT-A groups were described in Table 1. On the other hand, the two groups didn't differ in the mean number of monthly headache days, in the number of acute headache medications assumed, or for the percentage of $\mathrm{MOH}$. As well MIDAS and HAM-A scores were similar, as years of school education. For this reason, we used the significantly different parameters as covariates for PSM (see Table 1). After PSM, 36 matched couples of patients were selected. In this subset of patients, PSM succeeded to pair all covariates except age. Age resulted higher in OnaBoNT-A group being patients in average older by 7.67 years also after the trimming. The global course of the results didn't change. After the trimming, the analysis confirmed the results obtained on the original sample, with headache days reduction being significant at 180 days with a greater reduction in the STPP group and acute headache medications consumption being significantly greater in STPP at 90 and 180 days (see Table 2).

\section{Prediction of clinical responses}

DFA built three predictive models for the early clinical outcome: one with all data pooled together and one model for each group. The pooled model considered baseline number of headache days, HIT-6 score, HAM-A score, current prophylaxis and school years as predictors (see Table $3 \mathrm{~A}$ and Fig. 2A). In the corresponding plot all CR patients, but two, lie in the positive field of the Root 1 of the model, suggesting that Root 1 can discriminate between $\mathrm{CR}$ and the other classes of responses. No distinction is possible between PR and NR. In the OnaBoNT-A group (Table 3B and Fig. 2B), the model correctly predicted the outcome by using, in descending order, baseline number of headache days, HAM-A score, HIT-6 score, HAM-D score, and number of acute headache medications assumed at baseline. Also in this case all CR patients, except one, lie in the positive field of the Root 1, but moreover all, but one, PR patients are in the positive field of the Root 2 (upper part of the graphic) and $70 \%$ of NR patients are in the negative part of the Root 2 (lower part of the graphic). In the bottom left quadrant (the negative field of both roots) all patients are classified as NR. In the STPP group, the model was not significant (see Table $3 \mathrm{C}$ for details). In all predictive models (also in not significant model of STPP group) the most important variable accounting for the prediction was baseline number of headache days per month. When we compared the 
average value of baseline number of headache days, we found a higher number of headache days per month in NR, and intermediate number in PR and lower number in CR patients $(F=4.67 ; p=0.011)$. Posthoc comparison showed significant differences between NR and PR $(25.7 \pm 6.1$ vs. $22.7 \pm 6.1, p=0.007)$ and NR and CR ( $25.7 \pm 6.1$ vs. $21.7 \pm 7.4, p=0.04)$ (see Fig. 3 ). Regarding the 360 days response, only the model on the pooled data provided a significant result, showing that better predictors of late responses were the baseline number of acute headache medications and the level of school education (see Table 4 and Fig. 2D)

Table 4

DFA model of the 1 years response. The weight of variables on the prediction.

\begin{tabular}{|lll|}
\hline D. Pooled (late response) & Partial & p-level \\
\hline School years & $\mathbf{0 . 6 9 1}$ & $\mathbf{0 . 0 0 2}$ \\
\hline Age & 0.879 & 0.119 \\
\hline HA Days & 0.835 & 0.051 \\
\hline Chronicization years & 0.841 & 0.058 \\
\hline $\mathbf{N}^{\circ}$ analgesics & $\mathbf{0 . 8 2 3}$ & $\mathbf{0 . 0 4 0}$ \\
\hline HAM-D & 0.937 & 0.342 \\
\hline Curr. Prophyl. & 0.941 & 0.369 \\
\hline
\end{tabular}

\section{Discussion}

The present study yielded results on the comparison between OnaBoNT-A vs. STPP plus pharmacological therapy. We opted for a pragmatic-inspired observational design rather than a Randomized Clinical Trial (RCT) because it is not possible to provide a correct randomization in trials involving a psychotherapeutic approach, since acceptance of the psychotherapy treatment is the nominal requirement for it to succeed. Although RCTs remain the gold standard to test effectiveness of treatments, the real-world efficacy can differ from results obtained by RCTs. In this context real-world and pragmatic studies can provide information on the activity of established therapies also in the setting of routine clinical practice. According to a recent article by Ford, our observational study has some characteristics that are recommended for pragmatic trials [31]: patients were recruited amongst those who usually attended our Headache Center, therapeutic interventions were chosen by different doctors based on the therapeutic guidelines and patients profile. The recruited population was therefore the closest to real world CM population candidate to receive that treatment. Both OnaBoNT-A and STPP were delivered as usual, and regular 3-month follow-up visits were used to assess the clinical response, which is the routine follow-up schedule used in our outpatients clinic. 
Our study showed the overall beneficial effect on headache days and painkillers intake in OnaBoNT-A and the STPP-plus drug-of-choice groups with no difference at the last follow-up, after 12 months. Patients of the STPP plus drug group had less headache days at 3 months and less acute headache medications consumption at 3 and 6 months in both the original and PSM analyses. This suggests that on the long run the two treatments work equally well, but that STPP can have an added value in the short period. In line with this, the number of $\mathrm{MOH}$ patients decreased in a significant manner after 3 months only in the STPP group, according to previous data showing that STPP-associated treatment is more effective than pharmacological therapy alone at 6 and 12 months follow-up [11].

However, our study showed that STPP arm is concerned by a higher rate of dropouts, $78 \%$ (STPP) vs. $55 \%$ (OnaBoNT-A). Such rate of discontinuation is quite high but in line with recent data from the pharmacological arm of the FORWARD trial [32]. Interestingly, in the present study, the adherence dropped mostly at the time of the latest follow-up (12 months). A possible reason of the adherence in the OnaBoNT-A group could be related to the fact that, despite the global effect was similar in the two groups, OnaBoNT-A patients had a longer migraine and chronicization duration and therefore an improvement could have a more encouraging effect to sticking with the therapy as well as to the "invasive" nature of the OnaBoNT-A treatment. This pattern of dropping out could most likely reflect a failure of treatment efficacy with $\mathrm{CM}$ and $\mathrm{MOH}$ relapse and possibly in part the normal time-related turnover of attending $\mathrm{CM}$ patients.

From literature, we know that only $9 \%$ of patients drop and discontinue treatments for clinical improvement. We can speculate that a part of dropouts occurred for improvement, although it is likely that the larger part is due to therapy inefficacy. In our setting, in fact, both groups had the larger improvement early after the beginning of the two therapies. For STPP, it occurred just after STPP ending, when the number of headache days dropped by a third. Moreover, 8 patients became CR and didn't start pharmacological therapy throughout the entire follow-up. As well, OnaBoNT-A patients experienced the same response after the first administration. Both groups halved the painkillers consumption at this point. In part it could also depend from the advice to quit overuse [33,34] or as a direct consequence of the clinical improvement, but the fact that medical discontinuation was significantly higher in STPP suggests that psychotherapic intervention had an active role.

Since at baseline the two group differed for several demographic and clinical features (see Table 1 for details), we performed a PSM to mimic randomization in the two groups, as suggested in recent realworld studies $[35,36]$. In our sample, patients with a longer history and more lines of failed therapies were addressed to OnaBoNT-A and patients showing depressive symptoms (who later obtained higher scores in the HAM-D) to STPP. This is not surprising since enrolled patients should be those that most likely would receive that treatment in real life. However, after matching patients with the propensity score, results were highly confirmed, strengthening their robustness. We should acknowledge that, despite PSM, age remained different between groups. Instead of forcing the PSM to reach a better match, further reducing the number of patients, we preferred to repeat the analysis with this matching. This decision derived from two main reasons. First, PSM successfully resolved 4 out of 5 bias without changing results, 
so it is unlikely that pairing the age would modify the outcome. Second, we decided to have more than 30 subjects per group so that the number is high enough to compensate unpredictable variables that could alter the outcome, according to the central limit theory [37].

We also investigated the role of baseline characteristics in determining early and late response, obtaining significant results for the pooled data and OnaBoNT-A group. The number of headache days per month at baseline resulted the most important predictive factors for early response, and resulted proportional to the chance of response: NR had the higher baseline number of headache days, while PR patients had an intermediate value, and CR patients had the lowest one. It suggested that achieving a better outcome is easier in patients with less days of headache, suggesting that adequate treatment should be started before frequency of headaches becomes daily (more than 25 days/month).

Baseline painkillers consumption and the level of school education are the most important factors for the 12 months response. It is interesting to notice that 3- and 12-month predictors are different. Baseline analgesic consumption is probably related to an "addiction" profile that could reappear with time and easily facilitates medication overuse and migraine chronicization [38]. On the other hand, a higher level of school education is in general associated to a better outcome in psychotherapy studies [39].

\section{Conclusion}

In the long range, OnaBoNT-A monotherapy seems as effective as combined STTP plus pharmacological therapy in terms of headache burden reduction. STPP is more useful to induce an early discontinuation of medication overuse and can lower the number of headache days throughout the months. However, OnaBoNT-A treatment is related to a better long-term adherence to therapy so that it can be more reliable in granting a positive outcome. Moreover, for the early response, the DFA-based predictive model works better in OnaBoNT-A group than in the pooled data and in the STPP group. This latter result can pave the road to a first attempt of tailored therapy for CM patients with OnaBoNT-A.

\section{Abbreviations}

$\mathrm{CM}$ : chronic migraine, $\mathrm{MOH}$ : medication overuse headache, STPP: Short-term Psychodynamic Psychotherapy, OnaBonT-A: OnabotulinumtoxinA, PSM: Propensity score matching, DFA: Discriminant Function Analysis, PREEMPT: Phase III Research Evaluating Migraine Prophylaxis Treatment, BPI: Brief Psychodynamic Interview, HAM-D: Hamilton Rating Scale for Depression, HAM-A: Hamilton Rating Scale for Anxiety, CR: complete response, PR: partial response, NR: no response,

\section{Declarations}

\section{Ethics approval and consent to participate}

The study was approved by the Ethic Committee of the University Hospital Umberto I within the context of a larger study on predictive factors of response to migraine preventive therapies, and all patients 
participating in the study were asked to sign a written informed consent for the use of their clinical data.

\section{Consent for Publication}

Not applicable.

\section{Availability of data and materials}

Data are however available from the authors upon reasonable request and with permission of the Ethic Committee.

\section{Competing interests}

The authors declare that they have no competing interest.

\section{Funding}

Authors received no particular funding for this study.

\section{Authors' contributions}

MA, BP, AV and VPD designed the study; MA and BP and MP collected clinical data; BP perfomed BonT-A injections; RDS and CF performed statistical analysis; AV supervised statistical analysis; RDG and EG supervised STPP; AV and VDP supervised the study; all authors participated in results discussion and interpretation; MA and AV drafted the manuscript; BP, RDS and VDP revised the manuscript. All authors read and approved the final manuscript.

\section{Acknowledgements}

NA

\section{References}

1. Dodick DW (2006) Clinical practice. Chronic daily headache. N Engl J Med 354:158-165 
2. Diener HC, Holle D, Solbach K, Gaul C (2016) Medication-overuse headache: risk factors, pathophysiology and management. Nat Rev Neurol 12(10):575-583

3. Blumenfeld AM, Bloudek LM, Becker WJ et al (2013) Patterns of use and reasons for discontinuation of prophylactic medications for episodic migraine and chronic migraine: results from the second international burden of migraine study (IBMS-II). Headache 53(4):644-655

4. Buse DC, Manack A, Serrano D, Turkel C, Lipton RB (2010) Sociodemographic and comorbidity profiles of chronic migraine and episodic migraine sufferers. J Neurol Neurosurg Psychiatry 81(4):428-432

5. Ashina S, Serrano D, Lipton RB et al (2012) Depression and risk of transformation of episodic to chronic migraine. J Headache Pain 13(8):615-624. doi:. Epub 2012 Sep 25

6. Scher Al, Stewart WF, Ricci JA, Lipton RB (2003) Factors associated with the onset and remission of chronic daily headache in a population-based study. Pain 106(1-2):81-89

7. Orhurhu V, Olusunmade M, Akinola Y et al (2019) Depression Trends in Patients with Chronic Pain: An Analysis of the Nationwide Inpatient Sample. Pain Physician 22(5):E487-E494

8. Gaul C, Brömstrup J, Fritsche G, Diener HC, Katsarava Z (2011) Evaluating integrated headache care: a one-year follow-up observational study in patients treated at the Essen headache centre. BMC Neurol 11:124

9. Gaul C, Liesering-Latta E, Schäfer B, Fritsche G, Holle D (2016) Integrated multidisciplinary care of headache disorders: A narrative review. Cephalalgia 36(12):1181-1191

10. Ansari H, Ziad S (2016) Drug-Drug Interactions in Headache Medicine. Headache; 56(7):1241-8

11. Altieri M, Di Giambattista R, Di Clemente L et al (2009) Combined pharmacological and short-term psychodynamic psychotherapy for probable medication overuse headache: a pilot study. Cephalalgia;29(3):293-9

12. Grazzi L, Sansone E, Raggi A et al (2017) Mindfulness and pharmacological prophylaxis after withdrawal from medication overuse in patients with Chronic Migraine: an effectiveness trial with a one-year follow-up. J Headache Pain 18(1):15

13. Lilliengren $P$, Johansson R, Town JM, Kisely S, Abbass A (2017) Intensive Short-Term Dynamic Psychotherapy for generalized anxiety disorder: A pilot effectiveness and process-outcome study. Clin Psychol Psychother 24(6):1313-1321

14. Town JM, Abbass A, Driessen E, Luyten P, Weerasekera P (2017) Updating the Evidence and Recommendations for Short-Term Psychodynamic Psychotherapy in the Treatment of Major Depressive Disorder in Adults. Can J Psychiatry 62(1):73-74

15. Abbass A, Kisely S, Kroenke K (2009) Short-term psychodynamic psychotherapy for somatic disorders. Systematic review and meta-analysis of clinical trials. Psychother Psychosom 78(5):265274

16. Aurora SK, Dodick DW, Turkel CC et al (2010) OnabotulinumtoxinA for treatment of chronic migraine: results from the double-blind, randomized, placebo-controlled phase of the PREEMPT 1 trial. Cephalalgia 30(7):793-803 
17. Diener HC, Dodick DW, Aurora SK et al (2010) OnabotulinumtoxinA for treatment of chronic migraine: results from the double-blind, randomized, placebo-controlled phase of the PREEMPT 2 trial. Cephalalgia 30(7):804-814

18. Domínguez C, Pozo-Rosich P, Torres-Ferrús M et al (2018) OnabotulinumtoxinA in chronic migraine: predictors of response. A prospective multicentre descriptive study. Eur J Neurol 25(2):411-416

19. Negro A, Curto M, Lionetto L, Martelletti P (2015) A two years open-label prospective study of OnabotulinumtoxinA $195 \mathrm{U}$ in medication overuse headache: a real-world experience. $J$ Headache Pain 17:1

20. Andreou AP1,2, Al-Kaisy TM A et al (2018) Prospective real-world analysis of OnabotulinumtoxinA in chronic migraine post-National Institute for Health and Care Excellence UK technology appraisal. Eur J Neurol;25(8):1069-e83

21. Blumenfeld AM, Tepper SJ, Robbins LD et al (2019) Effects of onabotulinumtoxinA treatment for chronic migraine on common comorbidities including depression and anxiety. J Neurol Neurosurg Psychiatry 90(3):353-360

22. Gillieron E (1989) Short psychotherapeutic interventions (four sessions). Psychother Psychosom 51(1):32-37

23. Gilliéron E (1987) Setting and motivation in brief psychotherapy. Psychother Psychosom 47(2):105112

24. Eraksoy M, Butzkueven H, Ziemssen T, Zivadinov R (2014) Time for Change - Evolution of Realworld Evidence Outcome Measures in Multiple Sclerosis Exemplified by Fingolimod. Eur Neurol Rev 9(2):136-142

25. Rosenbaum PR, Rubin DB (1983) The Central Role of the Propensity Score in Observational Studies for Causal Effects. Biometrika 70(1):41-55

26. Austin PC (2011) An Introduction to Propensity Score Methods for Reducing the Effects of Confounding in Observational Studies. Multivariate Behav Res 46(3):399-424

27. Lachenbruch PA (1997) Discriminant diagnostics. Biometrics; 53: 1284-1292

28. De Sanctis R, Viganò A, Giuliani A et al (2018) Unsupervised versus Supervised Identification of Prognostic Factors in Patients with Localized Retroperitoneal Sarcoma: A Data Clustering and Mahalanobis Distance Approach. Biomed Res Int; 2018:2786163

29. De Sanctis R, Agostinetto E, Masci G et al (2018) Predictive Factors of Eribulin Activity in Metastatic Breast Cancer Patients. Oncology; 94 Suppl 1:19-28

30. Viganò A, Savastano E, Petolicchio B, Toscano M, De Sanctis R, Maestrini I, Di Piero V (2020) A Study of Clinical Features and Risk Factors of Self-Referring Emergency Department Headache Patients: A Comparison with Headache Center Outpatients. Eur Neurol 10:1-7

31. Ford I, Norrie J (2016) Pragmatic Trials. N Engl J Med 375(5):454-463

32. Rothrock JF, Adams AM, Lipton RB et al (2019) FORWARD Study: Evaluating the Comparative Effectiveness of OnabotulinumtoxinA and Topiramate for Headache Prevention in Adults With 
Chronic Migraine. Headache; 59(10):1700-1713

33. Rossi P, Faroni JV, Nappi G (2011) Short-term effectiveness of simple advice as a withdrawal strategy in simple and complicated medication overuse headache. Eur J Neurol 18(3):396-401

34. Rossi P, Faroni JV, Tassorelli C, Nappi G (2013) Advice alone versus structured detoxification programmes for complicated medication overuse headache $(\mathrm{MOH})$ : a prospective, randomized, openlabel trial. J Headache Pain 14:10

35. Prosperini L, Lucchini M, Haggiag $S$ et al (2018) Fingolimod vs dimethyl fumarate in multiple sclerosis: A real-world propensity score-matched study. Neurology 91(2):e153-e161

36. Kalincik T, Jokubaitis V, Spelman T et al (2018) Cladribine versus fingolimod, natalizumab and interferon $\beta$ for multiple sclerosis. Mult Scler 24(12):1617-1626

37. Pagano M, Gauvreau K (2000) Principles of Biostatistics. CRC, Cleveland

38. Ferraro S, Grazzi L, Muffatti R et al (2012) In medication-overuse headache, fMRI shows long-lasting dysfunction in midbrain areas. Headache. 2012 Nov-Dec;52(10):1520-34

39. Kim S, Park S (2014) The role of headache chronicity among predictors contributing to quality of life in patients with migraine: a hospital-based study. J Headache Pain 15:68

\section{Figures}




\section{A Headache days/month}

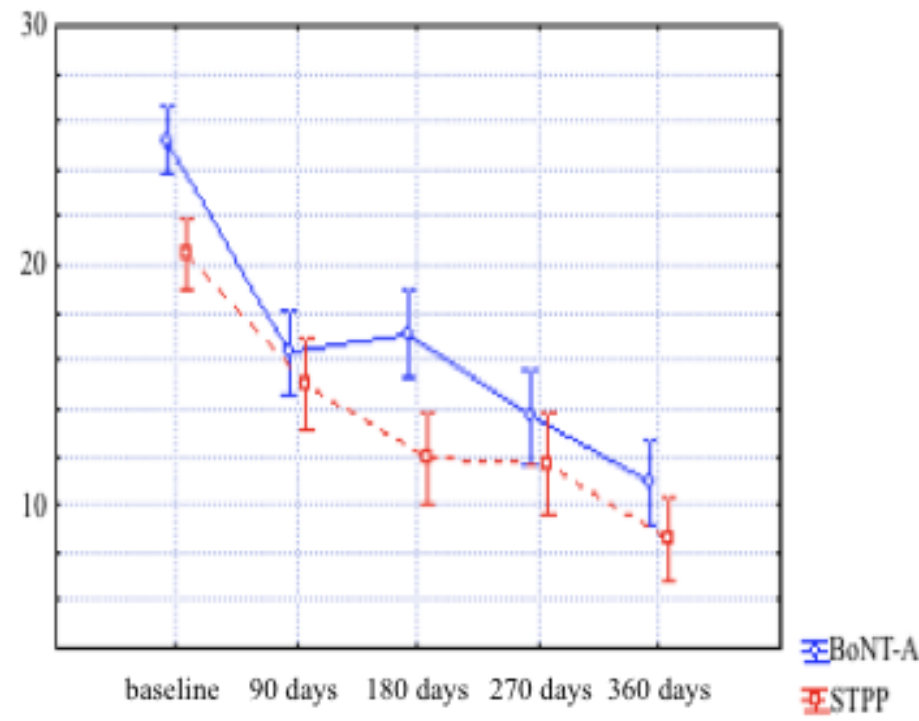

B $\quad \mathrm{N}^{\circ}$ analgesics/month

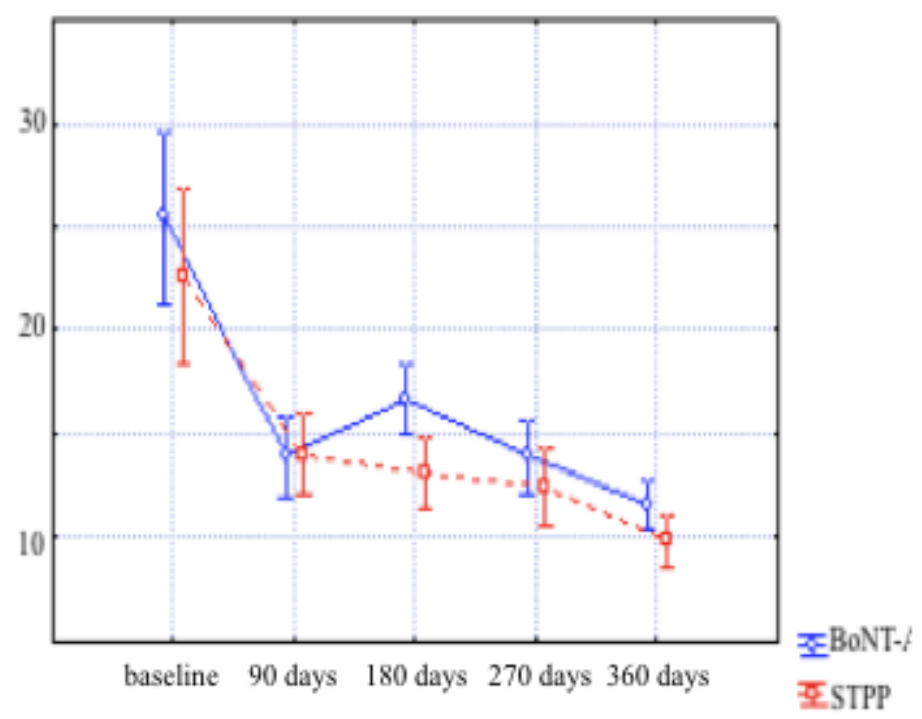

\section{Figure 1}

A-B. Clinical outcome of the two groups. In the panel A headache days reduction is shown, while analgesics consumption is shown in the panel $\mathrm{B}$. 
A

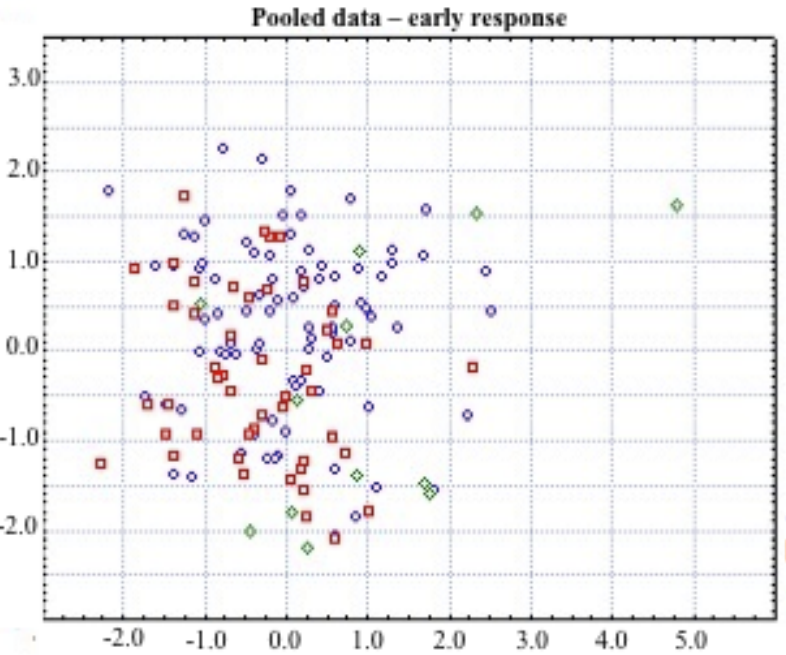

C

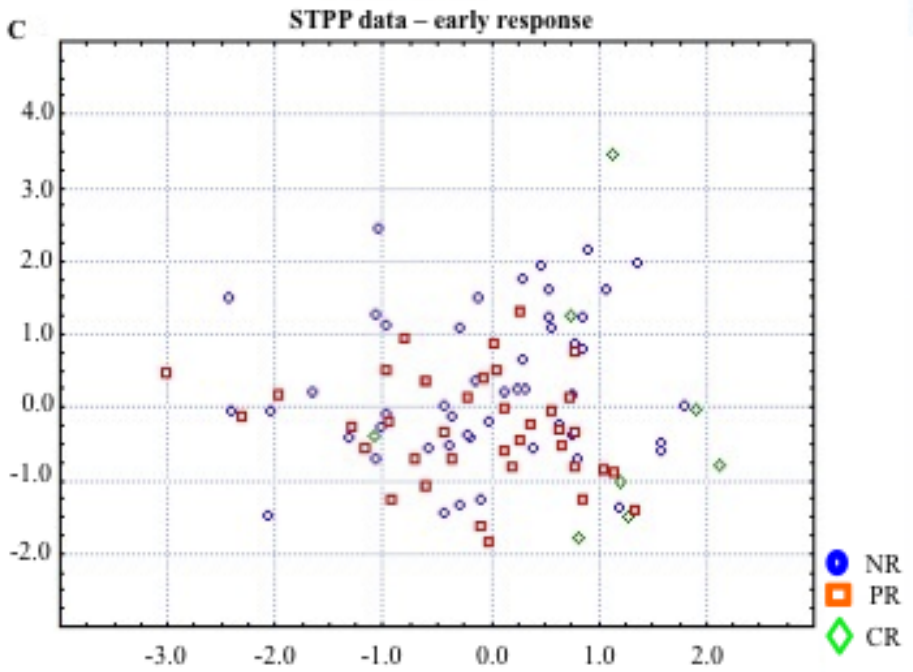

B
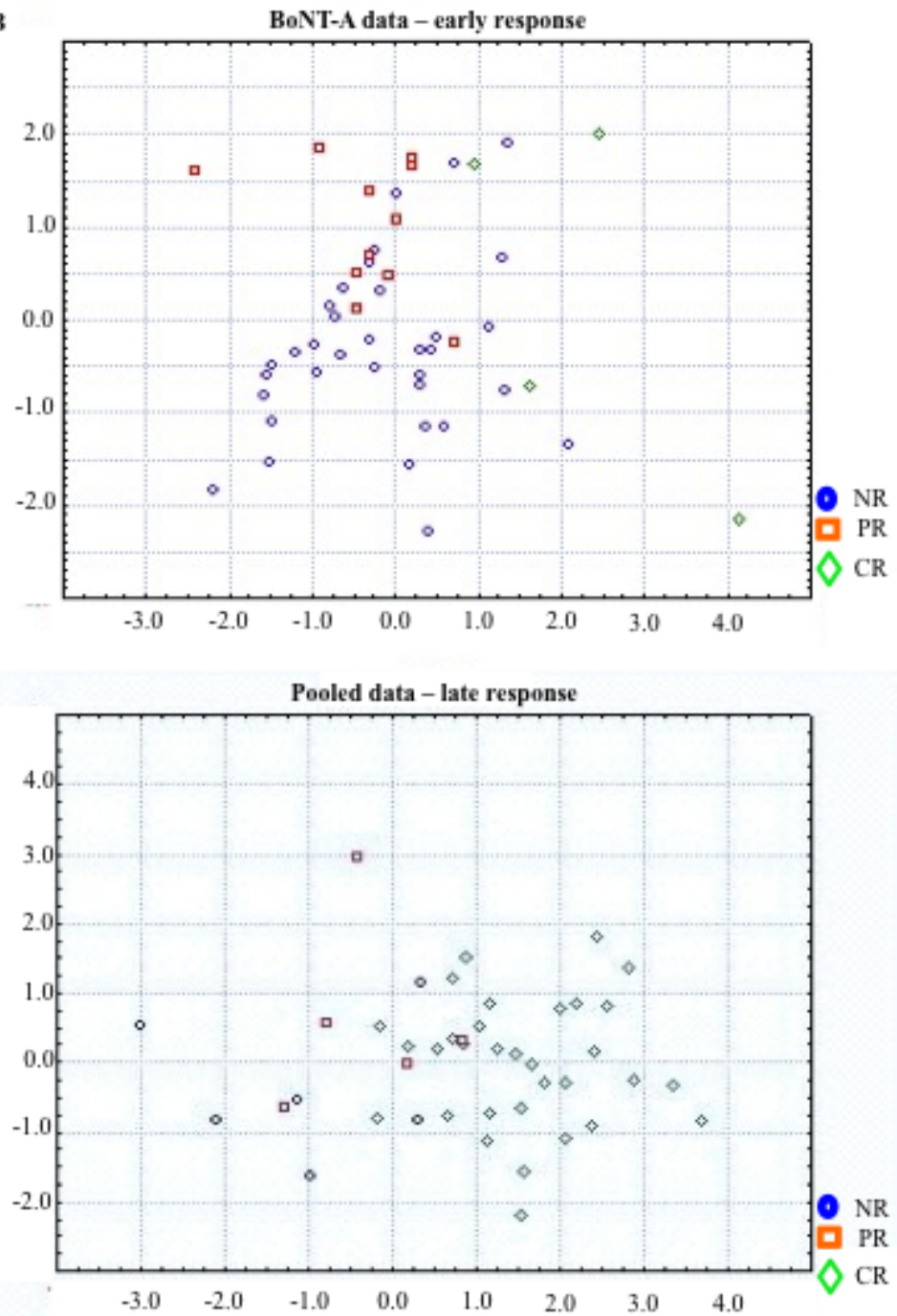

Figure 2

A-B-C-D Scatterplot of early and late response DFA classification for each patients. Data are from the pooled (A), OnaBoNT-A (B), STPP (C), and of late response from pooled data (D). In green CR, in red PR, and in blue NR. For a detailed legend of the figure, see supplementary materials. 


\section{Baseline headache days per month}

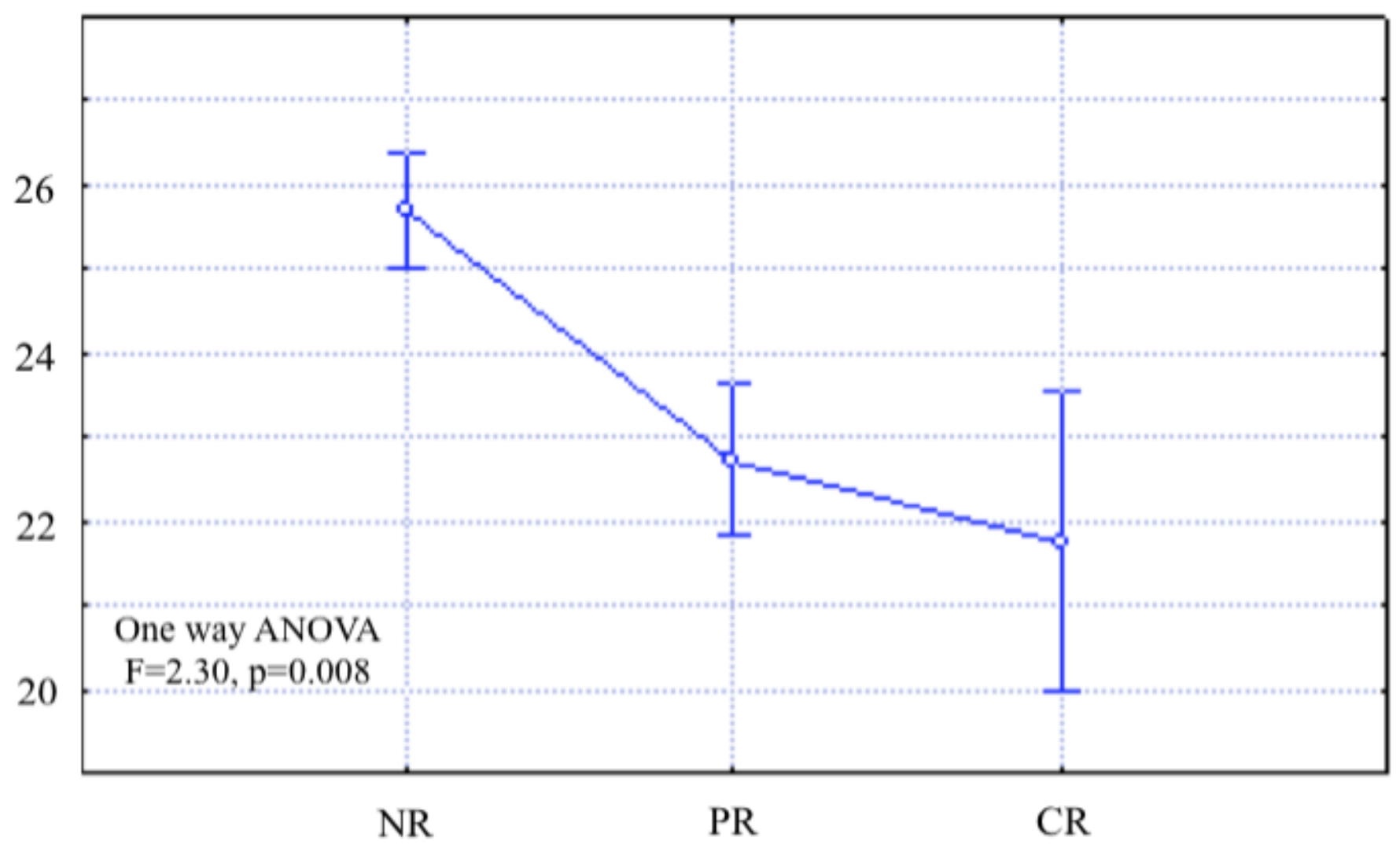

Figure 3

The average number of headache days at baseline. Circles represent mean values, and bars standard errors.

\section{Supplementary Files}

This is a list of supplementary files associated with this preprint. Click to download.

- Alessiani2020STROBEchecklistv4combined.doc

- Alessiani2020STROBEchecklistv4combined.doc 